\title{
Cathepsin B expression in tumour cells and laminin distribution in pulmonary adenocarcinoma
}

\author{
M Higashiyama, O Doi, K Kodama, H Yokouchi, R Tateishi
}

\begin{abstract}
Aims: To determine the correlation between cathepsin $B$ expression and laminin distribution in pulmonary adenocarcinoma tissue.

Methods: The distribution of cathepsin B and laminin was examined in $\mathbf{2 8}$ formalin fixed, paraffin wax embedded specimens of pulmonary adenocarcinoma tissue, using a double immunostaining technique with commercially available antibodies to cathepsin $B$ and laminin, respectively.

Results: Tumour cells in $23(82 \%)$ cases reacted to cathepsin $B$ : 13 cases were weakly positive and 10 were strongly positive. Laminin in tumour associated basement membrane produced various staining patterns: two cases had an almost continuous distribution of laminin in tumour associated basement membrane in the tumour tissues, while a moderately discontinuous laminin distribution pattern was found in 12 cases, and a highly fragmented pattern was found in 14 cases. The degree of cathepsin $B$ expression in tumour cells was significantly correlated with the break up of laminin staining. In some cases a discontinuous pattern of tumour associated laminin was frequently observed adjacent to cathepsin $B$ positive tumour cell nests.
\end{abstract}

Conclusions: Considering that cathepsin $B$ has the capacity to degrade basement membrane components, including laminin, the inverse correlation shown in this study between the increase in cathepsin $B$ expression by tumour cells and the diminution of laminin in tumour associated basement membrane could reflect local progression and spread by pulmonary adenocarcinoma.

(F Clin Pathol 1993;46:18-22)

Thoracic Surger

M Higashiyama

O Doi

K Kodama

H Yokouchi

Department of

Pathology

R Tateishi

The Centre for Adult

Diseases, Osaka,

Nakamichi 1-3-3,

Higashinuriku, Osaka

537, Japan

Correspondence to:

Dr M Higashiyama

Accepted for publication

14 May 1992 has the capacity to degrade laminin protein ${ }^{5}$ which forms part of the basement membrane, a thin sheet of extracellular matrix separating epitheiial cells from the underlying stroma. As en shown biochemically that cathep various types of neoplasms have been demonstrated to produce cathepsin $\mathrm{B},{ }^{6}{ }^{12}$ its role in neoplasms is now actively being investigated, with regard to the destruction of the extracellular matrix prior to neoplastic invasion and metastasis.

Recently, several human lung cancer cell lines have been shown to produce cathepsin B enzyme ${ }^{13}$ and we have shown, immunohistochemically, varying degrees of its expression in surgical specimens of pulmonary adenocarcinoma. ${ }^{14}$

\section{Methods}

Twenty eight patients with adenocarcinoma of the lung, who had been surgically treated in our institute, were studied. According to the international classification, ${ }^{15} 15$ patients were stage I, three were stage II, and 10 were stage IIIA. The adenocarcinoma was well differentiated in 14 patients, moderately differentiated in nine, and poorly differentiated in four.

Specimens prepared from resected tumour tissue, including the surrounding non-cancerous tissue, were fixed in formalin and embedded in paraffin wax. Each section was cut into $4 \mu \mathrm{m}$ thick slices. Sheep antisera to cathepsin B were purchased from The Binding Site Ltd, Birmingham, England, and rabbit antisera to laminin were purchased from Chemicon International Inc., West Temecula, California, USA.

Double immunostaining was performed in the same section, using the avidin-biotin complex peroxidase method to detect cathepsin B in the first step, and the avidin-biotin complexalkaline phosphatase method to detect laminin in the second step. In the first step each section was dewaxed in xylene and treated in methanol containing $1 \%$ hydrogen peroxide to inhibit endogenous peroxidase activity. After incubation with normal rabbit serum ( 1 in 50; Vector Laboratories Inc., Burlingame, California), anti-cathepsin B antibody (1 in 100) was applied to each section, which was then reacted with biotinylated rabbit anti-sheep gammaglobulin ( 1 in 200, Vector Laboratories Inc., Burlingame, California). After treatment with avidin-biotin peroxidase complex solution, the peroxidase reactions were visualised with $0.05 \%$ 3,3'-diaminobenzidine (DAB), containing $0.01 \% \mathrm{H}_{2} \mathrm{O}_{2}$ in TRIS-HCl-buffered solution $(\mathrm{pH} 7 \cdot 6)$. In the second step each section was incubated with $0.4 \%$ pepsin in $0.01 \mathrm{~N} \mathrm{HCl}$ 
Figure $1 A$

Immunostaining for cathepsin B in pulmonary adenocarcinoma tissue. Strongly positive staining with a fine granular

pattern is observed mainly in the cytoplasm of tumour cells (no counterstain).

\section{Figure $1 B$}

Immunostaining for laminin in pulmonary adenocarcinoma tissue. Laminin in tumour associated basement membrane is distributed in a moderately continuous pattern (arrow). As a positive control within the tissues, laminin is observed in the basement membrane surrounding the vascular structure (arrowhead) (no counterstain).
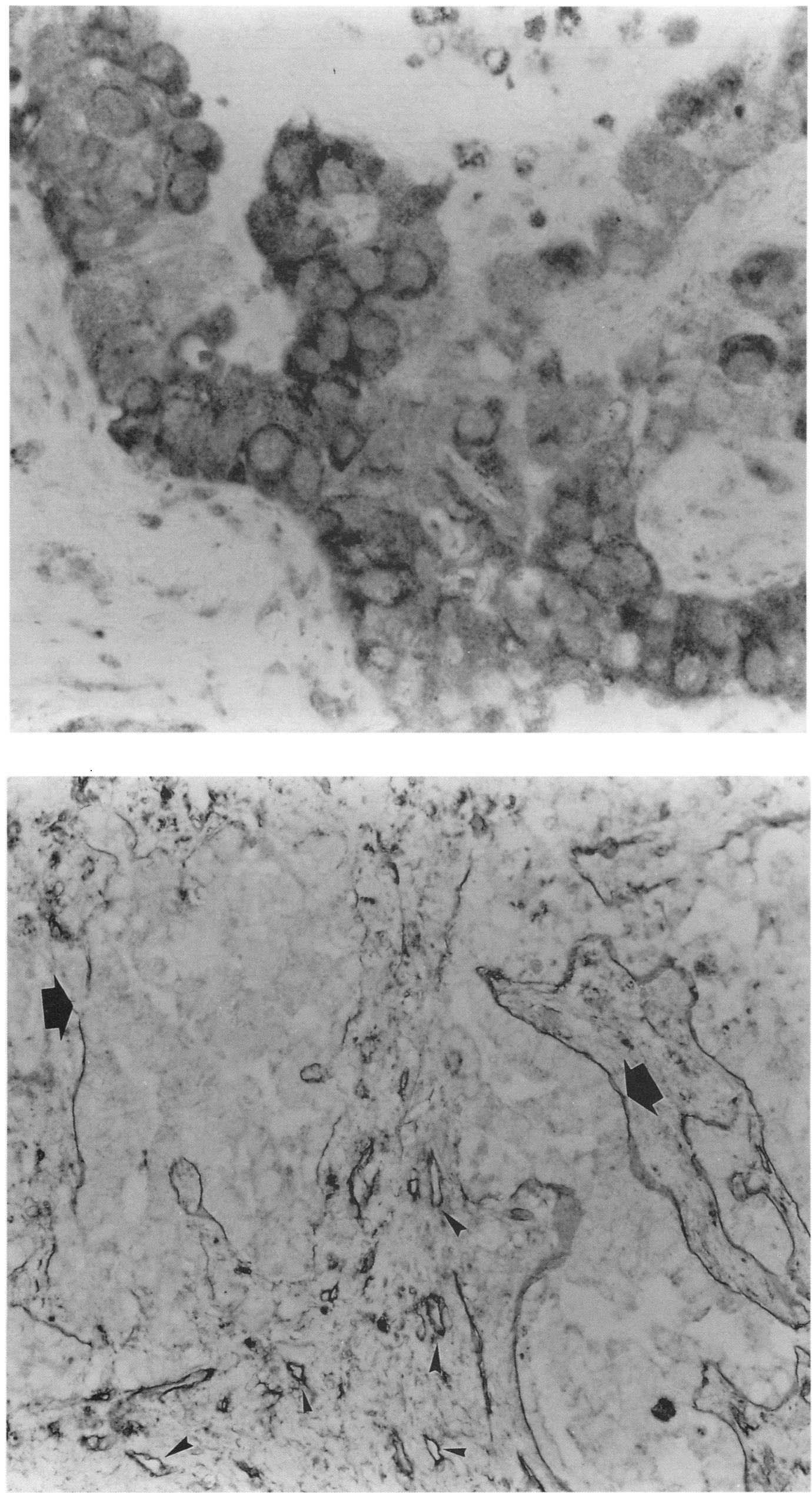
Correlation between cathepsin $B$ expression and laminin distribution in pulmonary adenocarcinoma tissue

\begin{tabular}{llll}
\hline & \multicolumn{2}{l}{ Cathepsin B expression } & \\
\cline { 2 - 4 } & & Positive & \\
\cline { 3 - 4 } & Negative $(n=5)$ & Weak $(n=13)$ & Strong $(n=10)$ \\
\hline $\begin{array}{l}\text { Laminin } \\
\text { Almost continuous } \mathrm{n}=2\end{array}$ & 2 & 0 & 0 \\
$\begin{array}{l}\text { Discontinuous } \\
\text { Moderate } \mathrm{n}=12\end{array}$ & 1 & & 2 \\
Fragmented $\mathrm{n}=14$ & 2 & 9 & 8 \\
\hline
\end{tabular}

p $<0.01$ : degree of cathepsin B expression $v$ laminin distribution pattern.

for one hour at $37^{\circ} \mathrm{C}$. Following treatment with normal goat serum ( 1 in 50), anti-laminin antibody ( 1 in 300) was applied. After the reaction with biotinylated goat antirabbit gammaglobulin ( 1 in 200), each section was treated with alkaline phosphatase-labelled streptavidin solution (Histofine SAB-AP Kit, Nichirei Inc., Tokyo). Alkaline phosphatase reactions were then detected by the HistoMark Blue test system (Kirkegaard \& Perry Laboratories Inc., Gaithersburg, Maryland), which includes the phosphate ester of 6-bromo-2-hydroxy3-naphthoic acid as buffered substrate solution, diazotised 4'-amino-2',5'-diethoxybenzanilide as Phthaloblue solution, and activator solution.

Staining results for cathepsin B detection were evaluated semiquantitatively, taking into account the percentage of cathepsin B positive cancer cells within the tumour tissues $(<10 \%$ negative $(-), 10 \%-80 \%$ weakly positive $(+)$, $>80 \%$ strongly positive $(++))$. Evaluation of tumour associated laminin expression was based on the laminin distribution pattern. This was classified into three types: almost continuous, moderately discontinuous, and highly fragmented, mainly within the pericentral, rather than the marginal areas of the tumour tissue, as heterogeneity of staining was noted in the same sections.

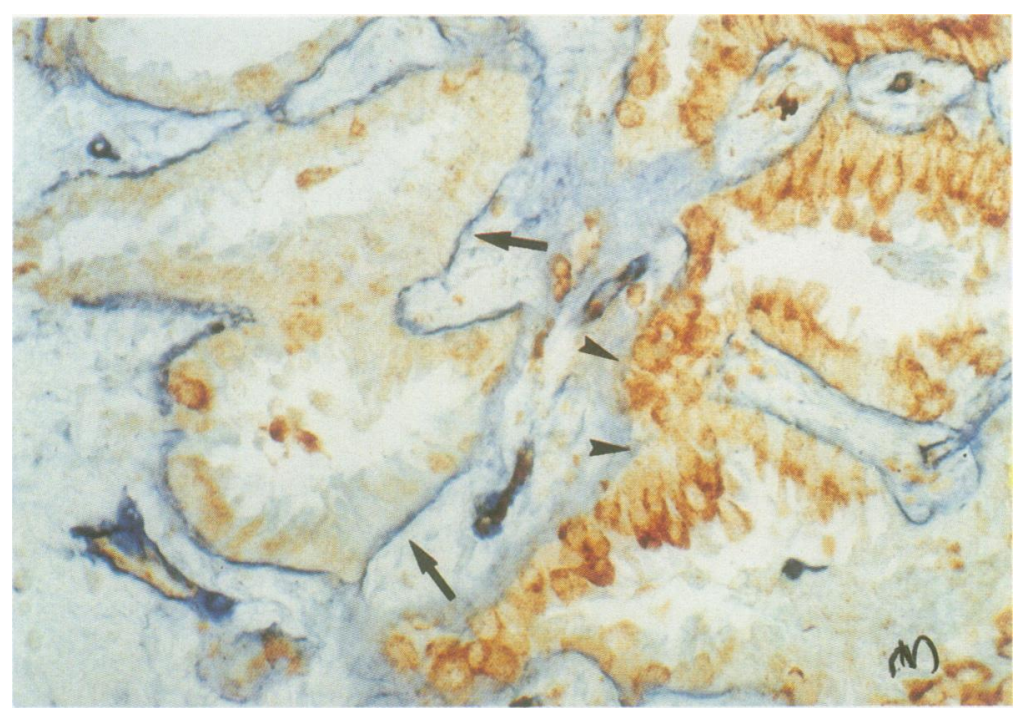

Figure 2 Double immunostaining for cathepsin $B$ and laminin. While cathepsin $B$ negative tumour cells or nests showed continuous distribution of laminin in tumour associated basement membrane (arrow), laminin has disappeared in the areas in contact with cathepsin B positive tumour cells (arrowhead) (no counterstain).
The results of the immunohistochemical study were evaluated using the $\chi^{2}$ test. A difference was considered to be significant when the $\mathrm{p}$ value was $<0.05$.

\section{Results}

The expression of cathepsin B and laminin expression in pulmonary adenocarcinoma tissues is shown in figs $1 \mathrm{~A}$ and B. Figure $1 \mathrm{~A}$ shows positive immunoreactivity for cathepsin B, with a diffuse or fine granular staining pattern, in the cytoplasm of some cancer cells, obtained in the first step of the double immunostaining method. Figure 1B shows laminin expression in tumour associated basement membrane, obtained only in the second step of this immunohistochemical method. As a positive control for laminin staining, laminin was usually detected as a distinct continuous pattern in the basement membrane bands underlying the respiratory and alveolar epithelia and surrounding the vascular structure within the same section.

Immunohistochemical analysis of the results of double immunostaining showed that 23 cases (13 weakly positive, 10 strongly positive) expressed cathepsin B in tumour cells, while five showed negative immunoreactivity for cathepsin B. In terms of laminin distribution, two cases showed distinct continuous, and focal although defective, laminin surrounding cancer cells; these were classified as almost continuous, while moderately discontinuous distribution of tumour-associated laminin was found in 12 cases, and highly fragmented or absent bands of laminin were observed in 14 cases.

The correlation between cathepsin B and tumour-associated laminin expression in lung adenocarcinoma tissues is shown in the table. The cathepsin $B$ negative cases exhibited almost continuous distribution of laminin. Overall, the more strongly cathepsin B was exhibited, the more significant was the discontinuous pattern of tumour-associated laminin $(p<0.01)$.

Furthermore, some cases showed that tumour-associated laminin was completely diminished in contact with cathepsin $B$ positive tumour cells, in contrast to the positive laminin expression in the neighbourhood of cathepsin B negative tumour cells (fig 2). However, these histological findings of an inverse correlation between cathepsin B and laminin distribution at the cellular level were not applicable in all the cases examined.

\section{Discussion}

Recent studies of cathepsin B activity not only in experimentally induced tumours, ${ }^{16-19}$ but also in human malignant tumour tissue, such as that of gastric, ${ }^{7}$ colorectal, ${ }^{910} 12$ breast, ${ }^{68}$ and lung cancer ${ }^{14}$ have shown that this enzyme is more active in tumours with a higher grade of malignant potential. In the studies of murine B16 melanoma cell lines ${ }^{17}{ }^{19}$ a highly metastatic cell variant showed increased cathepsin B activity, compared with a variant of low meta- 
static potential; and a significant increase in cathepsin B activity was found in human malignant lesions rather than in pre-malignant lesions. ${ }^{12}$ In pulmonary adenocarcinoma tissue we have shown that the postoperative survival of patients whose tissues were strongly cathepsin B positive was significantly lower than that of the less positive or negative patients. ${ }^{14} \mathrm{It}$ has therefore been suggested that cathepsin B in tumour cells may have a possible role in tumour progression, and that cathepsin B expression may be associated with malignant potential and may thus serve as a prognostic indicator.

Furthermore, as laminin is now considered to participate biologically in the attachment of epithelial cells to type IV collagen, it seems that tumour-associated laminin-that which is in contact with tumour cells or tumour cell nests-may participate in the attachment, spreading, and migration of tumour cells into the stroma. ${ }^{2121}$ Thus the degree of tumourassociated laminin expression or its distribution pattern in tumour tissues may be related to the malignant potential of the tumour; indeed, Nishino et $a l^{22}$ found that a discontinuous pattern of tumour-associated laminin in pulmonary adenocarcinoma, shown by the same immunohistochemical analysis as that used in the present study, may have been closely related to prognosis.

With regard to the action of cathepsin B in tumour progression, it has been proposed that this enzyme has the capacity to degrade such extracellular matrix components as collagen, fibronectin, proteoglycans, and elastin, as well as basement membrane components, including type IV collagen and laminin..$^{3-5116}$ In particular, since Lah et $a l^{5}$ biochemically demonstrated the proteolysis of laminin by cathepsin B, cathepsin B activity in tumour cells has been strongly linked with tumour malignancy. However, to our knowledge, a correlation between the distribution of cathepsin B and laminin in tumour-associated basement membrane in tumour tissues has not yet been reported on histological examination. Therefore, this immunohistochemical study may be the first report to show the inverse correlation of cathepsin B expression and laminin distribution pattern in such malignant tissues as pulmonary adenocarcinoma, on overall histological and cellular levels, using a double immunostaining technique.

Whether the discontinuous laminin pattern seen in tumour tissues might be a consequence of a decrease in laminin production or whether it might be a consequence of the degradation of tumour-associated basement membrane by tumour cells has not been known. From our present findings in pulmonary adenocarcinoma tissue, we emphasise that cathepsin B in tumour cells may destroy basement membrane, with a resulting diminution of laminin. ${ }^{11}$

Cathepsin B has recently been shown to consist of two types; one a 30-35 kilodalton precursor form in the lysosomal fraction, and the other a 20 kilodalton mature form in the plasma membrane fraction. ${ }^{1131923}$ The latter form in particular is considered to participate in the degradation of basement membrane components, indicating that the latter rather than the former form in tumour cells is more likely to be associated with tumour invasion and metastasis. In fact, an immunohistochemical study carried out by Erdel et al, using cancer cell lines, revealed that the expression of the latter form was distinct from that of the former. Using the same immunohistochemical methods as ours, Weiss et al, ${ }^{11}$ showed that cathepsin B was detected as a well defined granular pattern in the cytoplasm of noninvasive tumour cells; this pattern appeared to be present in lysosomes, while the pattern of cathepsin B expression in invasive tumour cells seemed to be less intense and more diffuse, suggesting that cathepsin $B$ may be redistributed to the plasma membrane. However, our observations showed that it appeared to be impossible to discriminate each type in the immunostained formalin fixed, paraffin wax embedded specimens in this study, in which the products of antigen-antibody reactions were mainly observed with a diffuse or fine granular pattern in the cytoplasm of tumour cells. Accordingly, it appears that the total of both cathepsin B types in tumour cells was evaluated in the present study.

Although cathepsin B expression was, overall, inversely correlated with laminin distribution in pulmonary adenocarcinoma tissue, some exceptions were observed: for instance, two cases with negative cathepsin B expression had a highly fragmented laminin distribution pattern. Distinct and continuous laminin distribution patterns were also observed in some areas of the pulmonary adenocarcinoma tissues despite strongly positive cathepsin B expression in tumour cells. Of two cases with negative cathepsin B expression and discontinuous laminin distribution, it can be speculated that cathepsin B had become unnecessary, because laminin degradation had already been completed, or that other proteinases, including cathepsin L, cathepsin D, and the metalloproteinase family, might act independently on laminin degradation. ${ }^{21} 24$

In conclusion, using an immunohistochemical technique, we showed an inverse correlation between cathepsin B in tumour cells and laminin expression in tumour-associated basement membrane in pulmonary adenocarcinoma tissue. These results suggest that cathepsin B in the tumour cells of pulmonary adenocarcinoma, via its proteolysis of laminin, may have an important role in tumour progression, in particular in local invasion and spread. In future, further immunohistochemical studies, specifically regarding cathepsin B in the plasma membrane of tumour cells, will be required to elucidate the possible role of this enzyme in tumour progression.

We thank $\mathrm{Y}$ Koyanagi and $\mathrm{Y}$ Funai for their laboratory assistance.

1 Greenbaum LM, Fruton JS. Purification and properties of beef spleen cathepsin B. $\mathcal{F}$ Biol Chem 1957;226:173-80. 2 Howie AJ, Burnett D, Crocker J. The distribution of cathepsin $B$ in human tissues. F Pathol 1985;145: 307-14. 
3 Katunuma N, Kominami E. Structures and functions of lysosomal thiol proteinases and their endogenous inhibilysosomal thiol proteinases and their end
tors. Curr Top Cell Regul 1983;22:71-101.

tors. Curr Top Cell Regul 1983;22:71-101.
4 Docherty K, Carrol RJ, Steiner DF. Conversion of proinsulin to insulin: Involvement of a 31,500 molecula weight thiol protease. Proc Natl Acad Sci USA 1982, 79:4613-7

5 Lah TH, Buck MR, Honn KV, et al. Degradation of laminin by human tumor cathepsin B. Clin Exp Metastasis 1989;7:461-8.

6 Poole AR, Tiltman KJ, Recklies AD, Stocker TAM. Dif ferences in secretion of the proteinase cathepsin B at the edges of human breast carcinomas and fibroadenomas. Nature 1978;273:545-7.

7 Watanabe $\mathrm{M}$, Higashi $\mathrm{T}$, Hashimoto $\mathrm{M}$, et al. Elevation of tissue cathepsin $B$ and $\mathrm{L}$ activities in gastric cancer. tissue cathepsin $\mathrm{B}$ and $\mathrm{L}$ activitienterol $1987 ; 34: 120-2$.

8 Krepela E, Bartek J, Skalkova D. Cytochemical and biochemical evidence of cathepsin B in malignant transformed and normal breast epithelial cells. $f$ Cell Sci transformed and

9 Sheahan K, Shuja S, Murnane MJ. Cysteine protease activities and tumor development in human colorectal carcinoma. Cancer Res 1989;49:3809-14.

10 Maciewicz RA, Wardale RJ, Etherington DJ, Paraskeva C. Immunodetection of cathepsin $B$ and $L$ present in and secreted from human pre-malignant and malignant colorectal tumor cell lines. Int $\mathcal{F}$ Cancer 1989;43:478-86.

11 Weiss RE, Liu BC-S, Ahlering T, Dubeau L, Droller MJ. Mechanisms of human bladder tumor invasion: role of protease cathepsin. Br $\mathcal{F}$ Urol 1990;144:798-804.

12 Shuja S, Sheahan K, Murnane MJ. Cysteine endopeptidase and carcinomas. Int $\mathcal{f}$ Cancer 1991;49:341-6.

13 Erdel M, Trefz G, Spiess E, et al. Localization of cathepsin $\mathrm{B}$ in two human lung cancer cell lines. $\mathrm{f}$ Histochem B in two human lung cance
Cytochem 1990;38:1313-21.
14 Higashiyama M, Doi O, Kodama K, Yokouchi H, Tateishi R. The prognostic implications of the cathepsin $B$ expression in pulmonary adenocarcinomas. An immunohisto37:1151-7.

15 Moutain CF. A new international staging system for lung cancer. Chest 1986;89:225-33.

16 Graf M, Baici A, Strauli F. Histochemical localization of cathepsin $B$ at the invasion front of the rabbit V2 carcinoma. Lab Invest 1981;45:587-96.

17 Sloane BF, Honn KV, Sadler JG, et al. Cathepsin B activity in B16 melanoma cells: a possible marker for metastatic potential. Cancer Res 1982;42:980-6.

18 Koppel P, Baici A, Keist R, Matzku S, Keller R, Cathepsin B-like proteinase as a marker for metastatic tumor cell variants. Exp Cell Biol 1984;52:293-9.

19 Rozhin J, Robinson D, Stevens MA, et al. Properties of a plasma membrane-associated cathepsin B-like cysteine plasma membrane-associated cathepsin B-like cysteine Res 1987;47:6620-8.

20 Fligiel SEG, Rodrigues AF, Knibbs RN, McCoy J, Varani J. Characterization of laminin-stimulated adherent and motility in tumor cell. Oncology 1985;42:265-71.

21 Starkey JR. Cell-matrix interaction during tumor invasion. Cancer Metastasis Rev 1990;9:113-23.

22 Nishino T, Ishida T, Oka T, Yasumoto K, Sugimachi $\mathrm{K}$. Prognostic significance of laminin in adenocarcinoma of the lung. F Surg Oncol 1990;43:214-8.

23 Sloane BF, Rozhin J, Johnson K, et al. Cathepsin B: Association with plasma membrane in metastatic tumors. Proc Natl Acad Sci USA 1986;83:2483-7.

24 Zucker S, Lysik RM, Wieman J, Wikie DP, Lane B. Diversity of human pancreatic cancer cell proteinases: Role of cell membrane metalloproteinases in collagenolysis and cytolysis. Cancer Res 1985;45:6168-78. 